\title{
Use of the van Cittert-Zernike theorem for partially polarized sources
}

\author{
Franco Gori and Massimo Santarsiero \\ Dipartimento di Fisica, Università Roma Tre, and Istituto Nazionale per la Fisica della Materia, Via della Vasca Navale 84, \\ I-00146 Rome, Italy
}

\section{Riccardo Borghi}

Dipartimento di Ingegneria Elettronica, Università Roma Tre, and Istituto Nazionale per la Fisica della Materia, Via della Vasca Navale 84, I-00146 Rome, Italy

\section{Gemma Piquero}

Departmento de Óptica, Universidad Complutense de Madrid, Ciudad Universitaria s/n, E-28040 Madrid, Spain

Received May 15, 2000

\begin{abstract}
We introduce an extension of the van Cittert-Zernike theorem to spatially incoherent sources with partial polarization. We show through a simple example that fields generated by such sources can possess correlation matrices with interesting properties. In particular, we show that by suitable modulation of the polarization state across the incoherent source, the correlation between the orthogonal components of the field as well as the degree of polarization may drastically change on propagation. (C) 2000 Optical Society of America

OCIS codes: $030.0030,060.2380,230.6080,260.5430$.
\end{abstract}

There is currently an interest in optical beams that are both partially coherent from the spatial standpoint and partially polarized..$^{1-4}$ Such beams can be described by an approximate version of the general tensorial theory of the electromagnetic field developed by Wolf. ${ }^{5}$ In fact, a single $2 \times 2$ matrix, called a beam coherencepolarization (BCP) matrix, is sufficient to yield a complete account of the second-order statistical properties of the field. ${ }^{3}$ In scalar coherence theory, the van Cittert-Zernike theorem is a fundamental tool for studying the propagation processes of partially coherent fields. ${ }^{5,6}$ Moreover, several partially coherent beams are generated starting from a primary spatially incoherent source. ${ }^{5,7,8}$ In such synthesis procedures the van Cittert-Zernike theorem plays a major role. We have observed that a spatially incoherent source can exhibit partial polarization and that the polarization state can change from one point to another across the source. Thus a suitable extension of the van Cittert-Zernike theorem to these sources should be sought. In this Letter we introduce such an extended version of the theorem. We then work out a specific example. It will be shown that the correlation functions appearing in the BCP matrix can behave in a rather different way with respect to each other. This will help the reader to appreciate the significance of the vectorial case. Furthermore, our results suggest that in the vectorial case, too, the van Cittert-Zernike theorem can serve as a useful tool in synthesis processes.

We use a reference frame in which the $z$ axis coincides with the mean direction of propagation of the beam. At a typical transverse plane, the vector $\mathbf{r}$ is used to specify the position of a point. Let us recall that, for a quasi-monochromatic field, the BCP matrix is defined $\mathrm{as}^{3}$

$$
\hat{J}\left(\mathbf{r}_{1}, \mathbf{r}_{2}, z\right)=\left[\begin{array}{cc}
J_{x x}\left(\mathbf{r}_{1}, \mathbf{r}_{2}, z\right) & J_{x y}\left(\mathbf{r}_{1}, \mathbf{r}_{2}, z\right) \\
J_{y x}\left(\mathbf{r}_{1}, \mathbf{r}_{2}, z\right) & J_{y y}\left(\mathbf{r}_{1}, \mathbf{r}_{2}, z\right)
\end{array}\right]
$$

where

$$
\begin{array}{r}
J_{\alpha \beta}\left(\mathbf{r}_{1}, \mathbf{r}_{2}, z\right)=\left\langle E_{\alpha}{ }^{*}\left(\mathbf{r}_{1}, z ; t\right) E_{\beta}\left(\mathbf{r}_{2}, z ; t\right)\right\rangle, \\
\alpha, \beta=x, y .
\end{array}
$$

The angle brackets denote time average, and $E_{\alpha}(\alpha=$ $x, y)$ is a Cartesian component of the time-dependent electric field.

In the framework of scalar theory a spatially incoherent source is characterized by means of a $\delta$-like mutual intensity function, which expresses the fact that the fields at any two distinct points across the source plane are uncorrelated. ${ }^{5}$ In the same way we define a partially polarized, spatially incoherent source as one whose BCP matrix elements $J_{\alpha \beta}$ have the form

$$
J_{\alpha \beta}\left(\boldsymbol{\rho}_{1}, \boldsymbol{\rho}_{2}, 0\right)=l^{2} I_{0 \alpha \beta}\left(\boldsymbol{\rho}_{1}\right) \delta\left(\boldsymbol{\rho}_{2}-\boldsymbol{\rho}_{1}\right),
$$

where $\delta$ is the two-dimensional Dirac function, $l$ is a constant with the dimension of length, and $I_{0 \alpha \beta}$ is a function, possibly a complex one when $\alpha \neq \beta$, whose modulus with the dimension of intensity. To a good approximation, we can equate $l$ with the mean wavelength of the radiation. ${ }^{9}$ Accordingly, we let $l=\lambda$.

We denote as $K\left(\mathbf{r}_{1}, \boldsymbol{\rho}_{1}, z\right)$ the kernel of propagation from the plane $z=0$ to the plane $z=$ constant $>0{ }^{6}$ 
Each element of the BCP matrix obeys a propagation formula of the form ${ }^{10}$

$$
\begin{array}{r}
J_{\alpha \beta}\left(\mathbf{r}_{1}, \mathbf{r}_{2}, z\right)=\iint J_{0 \alpha \beta}\left(\boldsymbol{\rho}_{1}, \boldsymbol{\rho}_{2}\right) K^{*}\left(\mathbf{r}_{1}, \boldsymbol{\rho}_{1}, z\right) \\
\times K\left(\mathbf{r}_{2}, \boldsymbol{\rho}_{2}, z\right) \mathrm{d}^{2} \rho_{1} \mathrm{~d}^{2} \rho_{2}, \\
\alpha, \beta=x, y .
\end{array}
$$

On inserting Eq. (3) into Eq. (4), we can evaluate the BCP matrix of the propagated field.

For beamlike fields the propagation kernel can be written in the paraxial approximation as

$$
K(\mathbf{r}, \boldsymbol{\rho}, z)=\frac{-i \exp (i k z)}{\lambda z} \exp \left[\frac{i k}{2 z}(\mathbf{r}-\boldsymbol{\rho})^{2}\right]
$$

where $k=2 \pi / \lambda$. When Eqs. (3) and (5) are inserted into Eq. (4), the result is

$$
\begin{aligned}
& J_{\alpha \beta}\left(\mathbf{r}_{1}, \mathbf{r}_{2}, z\right)=\frac{\exp \left[\frac{i k}{2 z}\left(r_{2}{ }^{2}-r_{1}{ }^{2}\right)\right]}{z^{2}} \\
& \times \int I_{0 \alpha \beta}(\boldsymbol{\rho}) \exp \left[\frac{-2 \pi i}{\lambda z} \boldsymbol{\rho} \cdot\left(\mathbf{r}_{2}-\mathbf{r}_{1}\right)\right] \mathrm{d}^{2} \rho,
\end{aligned}
$$

which represents the extension of the van Cittert-Zernike theorem to partially polarized beams. It should be stressed that Eq. (6) is also valid in the near field. In the following we limit ourselves to the far-zone region, where the quadratic phase factor in front of the integral can be approximated as 1 .

We can put into evidence some consequences of the theorem by means of a simple example. Let us start from a spatially incoherent and unpolarized source. For simplicity we refer to a two-dimensional case, in which one of the transverse coordinates can be neglected. Then the BCP matrix can be written as

$$
\hat{J}_{\text {in }}\left(\xi_{1}, \xi_{2}, 0\right)=\lambda I_{0} \delta\left(\xi_{2}-\xi_{1}\right)\left[\begin{array}{ll}
1 & 0 \\
0 & 1
\end{array}\right],
$$

where $I_{0}$ is a constant-intensity factor and $\xi$ denotes an abscissa across the source plane (see Fig. 1).

To endow the source with polarization properties we use a linear polarizer. More specifically, we cover the source with a single period of a linear polarization grating. ${ }^{11-14}$ In such a grating the angle, say $\theta$, between the transmission axis and the $\xi$ axis is a linear function of $\xi$ of the form $\theta=\pi \xi / L, L$ being the period.

The BCP matrix just beyond this polarizer can be calculated $\mathrm{as}^{3}$

$$
\begin{aligned}
\hat{J}_{\text {out }}\left(\xi_{1}, \xi_{2}, 0\right)= & \hat{T}^{\dagger}\left(\xi_{1}\right) \hat{J}_{\text {in }}\left(\xi_{1}, \xi_{2}, 0\right) \hat{T}\left(\xi_{2}\right) \\
& \times \operatorname{rect}\left(\xi_{1} / L\right) \operatorname{rect}\left(\xi_{2} / L\right),
\end{aligned}
$$

where $\operatorname{rect}(u)=1$ for $|u| \leq 1 / 2$ and 0 otherwise. Furthermore, the dagger denotes the Hermitian conjugate, and

$$
\begin{aligned}
& \hat{T}=\left[\begin{array}{cc}
C^{2} & C S \\
C S & S^{2}
\end{array}\right], \\
& C=\cos \pi \xi / L, \quad S=\sin \pi \xi / L
\end{aligned}
$$

is the Jones matrix of the polarizer. ${ }^{3}$

Since the field specified by Eqs. (8) and (9) is linearly polarized, there is a perfect correlation between any two field components at a typical source point.

When we substitute Eqs. (8) and (9) into the twodimensional version of Eq. (6), the normalized elements $^{3}$ of the BCP matrix at the far zone turn out to be

$$
\begin{aligned}
j_{x x}\left(x_{1}, x_{2}, z\right)= & \operatorname{sinc}(L \nu)+\frac{1}{2} \operatorname{sinc}(L \nu-1) \\
& +\frac{1}{2} \operatorname{sinc}(L \nu+1), \\
j_{y y}\left(x_{1}, x_{2}, z\right)= & \operatorname{sinc}(L \nu)-\frac{1}{2} \operatorname{sinc}(L \nu-1) \\
& -\frac{1}{2} \operatorname{sinc}(L \nu+1), \\
j_{x y}\left(x_{1}, x_{2}, z\right)= & \frac{1}{2 i}[\operatorname{sinc}(L \nu-1) \\
& -\operatorname{sinc}(L \nu+1)],
\end{aligned}
$$

where $\nu=\left(x_{2}-x_{1}\right) /(\lambda z)$ and $\operatorname{sinc}(u)$ stands for $\sin (\pi u) /(\pi u)$.

The scalar-equivalent mutual intensity ${ }^{3}$ is the sum of the diagonal elements of the BCP matrix and in normalized form has the expression

$$
j_{\mathrm{eq}}\left(x_{1}, x_{2}, z\right)=\operatorname{sinc}(L \nu)
$$

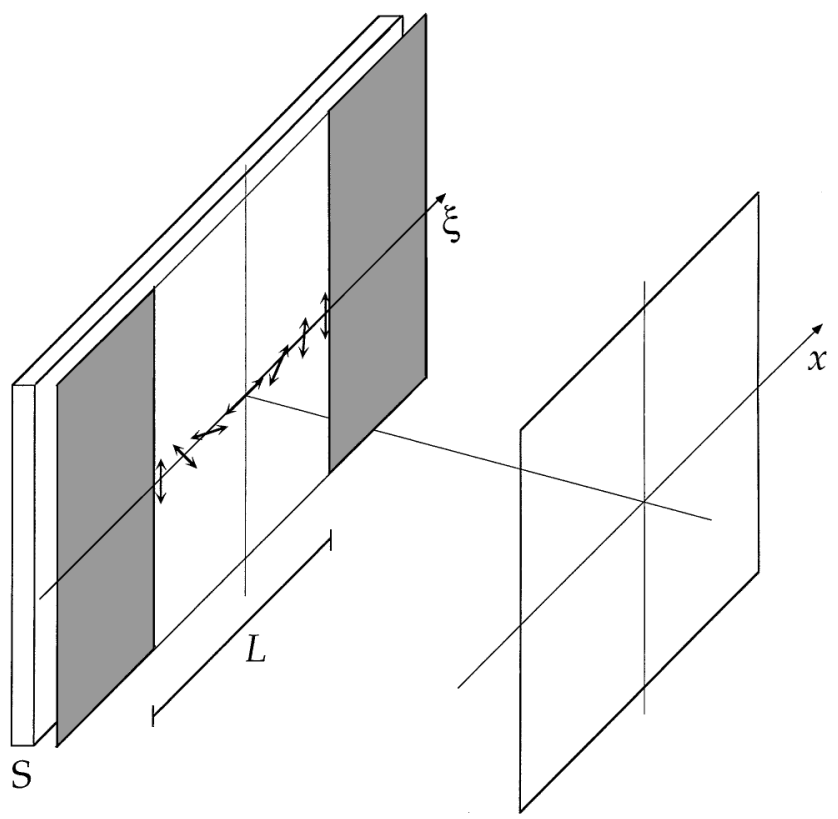

Fig. 1. Notation used in the evaluation of the BCP matrix for the field generated by the incoherent unpolarized primary source $(\mathrm{S})$ covered by a polarizing filter. 


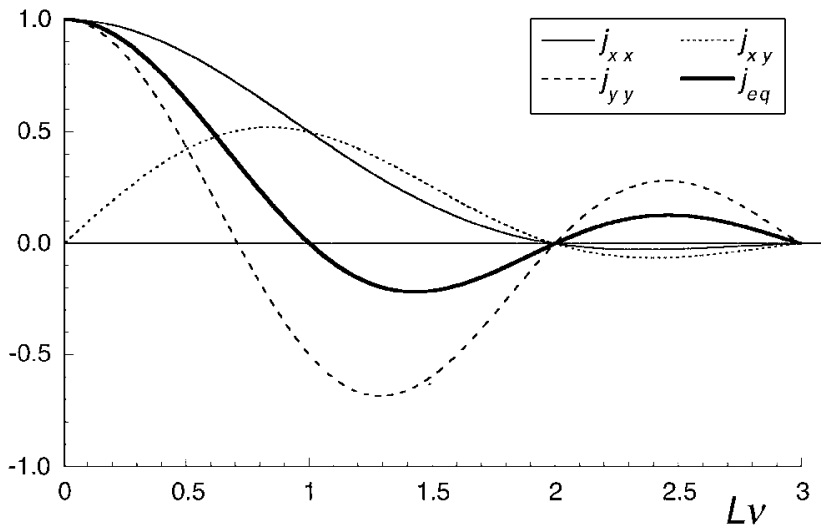

Fig. 2. Normalized elements of the BCP matrix versus $L \nu$, where $L$ is the width of the polarizer and $\nu=\left(x_{2}-\right.$ $\left.x_{1}\right) / \lambda z$. The scalar-equivalent degree of coherence, $j_{\text {eq }}$, is shown by the thicker solid curve.

Plots of the functions (10) and (11) are shown in Fig. 2. It can be seen from the figure that the well-known property $^{6}$ according to which light generated by a spatially incoherent source acquires coherence in the course of propagation is shared by the vectorial results. However, the behaviors of the correlation functions for the $x$ and $y$ components of the electric field are different, and both of them differ from the scalar prediction [Eq. (11)]. Further, an intriguing behavior is exhibited by $j_{x y}$. In fact, there is no correlation between the $x$ and $y$ components of the field at the same spatial point, whereas a significant correlation between the same components is exhibited at two suitably chosen distinct points. This result should be contrasted with the situation at the source, at which there is a perfect local correlation and no correlation at all between two field components at distinct points. We can then see that the BCP matrix can reveal physical features that would not be appreciated in the scalar realm. In the present case all the vectorial aspects would be hidden behind the familiar Eq. (11). Further, the degree of polarization $P$ can be evaluated through ${ }^{5}$

$$
P(x, z)=\left[\frac{\left(J_{x x}-J_{y y}\right)^{2}+4\left|J_{x y}\right|^{2}}{\left(J_{x x}+J_{y y}\right)^{2}}\right]^{1 / 2},
$$

where each $J_{\alpha \beta}$ element has to be evaluated for $x_{1}=$ $x_{2}=x$. It follows from Eqs. (10) that for $x_{1}=x_{2}$ the normalized off-diagonal element $j_{x y}$ vanishes, whereas the diagonal elements are equal to each other. The same occurs for the unnormalized $J_{\alpha \beta}$ elements. Accordingly, the degree of polarization equals 0 . This means that light that emerges from the source in a state of nonuniform complete linear polarization becomes completely unpolarized on propagation.

Finally, we note that the functions appearing in Eqs. (10) depend on $x_{1}-x_{2}$ only. This suggests that the extended van Cittert-Zernike theorem can be the basis for procedures aimed at synthesizing partially polarized Schell model sources in much the same way as is done in the scalar case. ${ }^{15}$ As demonstrated by our example, a significant role in this synthesis process can be played by polarization gratings, which are currently being investigated for many purposes. ${ }^{11-14}$

G. Piquero acknowledges support from the the Spanish project PB97-0295 and the program Becas Internacionales Universidad Complutense/Flores Valles. F. Gori's e-mail address is gori@uniroma1.it.

\section{References}

1. D. F. V. James, J. Opt. Soc. Am. A 11, 1641 (1994).

2. R. Martínez-Herrero, P. M. Mejías, and J. M. Movilla, Opt. Lett. 22, 206 (1997).

3. F. Gori, M. Santarsiero, S. Vicalvi, R. Borghi, and G. Guattari, J. Eur. Opt. Soc. A Pure Appl. Opt. 7, 941 (1998).

4. S. R. Seshadri, J. Opt. Soc. Am. A 16, 1373 (1999).

5. See, for example, L. Mandel and E. Wolf, Optical Coherence and Quantum Optics (Cambridge U. Press, Cambridge, 1995).

6. M. Born and E. Wolf, eds., Principles of Optics, 7th ed. (Cambridge U. Press, Cambridge, 1999).

7. P. de Santis, F. Gori, G. Guattari, and C. Palma, Opt. Commun. 29, 256 (1979).

8. J. Turunen, A. Vasara, and A. T. Friberg, J. Opt. Soc. Am. A 8, 282 (1991).

9. J. W. Goodman, Statistical Optics (Wiley, New York, 1985).

10. F. Gori, M. Santarsiero, R. Borghi, and G. Guattari, Opt. Commun. 163, 159 (1999).

11. L. Nikolova, M. Ivanov, T. Todorov, and S. Stoyanov, Bulg. J. Phys. 20, 46 (1993).

12. P. Rochon, V. Drnoyan, and A. Natansohn, Proc. SPIE 3491, 306 (2000).

13. F. Gori, Opt. Lett. 24, 584 (1999).

14. C. G. Someda, Opt. Lett. 25, 1657 (2000).

15. P. De Santis, F. Gori, and G. Guattari, J. Opt. Soc. Am. A 3, 1258 (1986). 